\title{
National Tuberculosis Genotyping and Surveillance Network: Design and Methods
}

\author{
Jack T. Crawford, ${ }^{*}$ Christopher R. Braden, ${ }^{*}$ Barbara A. Schable, ${ }^{*}$ and Ida M. Onorato*
}

\begin{abstract}
The National Tuberculosis Genotyping and Surveillance Network was established in 1996 to perform a 5year, prospective study of the usefulness of genotyping Mycobacterium tuberculosis isolates to tuberculosis control programs. Seven sentinel sites identified all new cases of tuberculosis, collected information on patients and contacts, and obtained patient isolates. Seven genotyping laboratories performed DNA fingerprinting analysis by the international standard IS6110 method. Biolmage Whole Band Analyzer software was used to analyze patterns, and distinct patterns were assigned unique designations. Isolates with six or fewer bands on IS6110 patterns were also spoligotyped. Patient data and genotyping designations were entered in a relational database and merged with selected variables from the national surveillance database. In two related databases, we compiled the results of routine contact investigations and the results of investigations of the relationships of patients who had isolates with matching genotypes. We describe the methods used in the study.
\end{abstract}
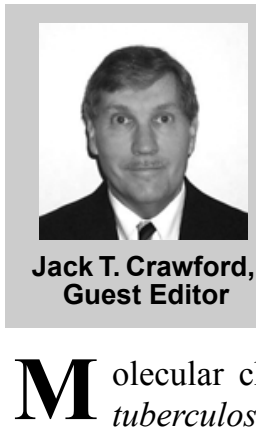

Dr. Crawford is chief of the Immunology and Molecular Pathogenesis Section, Tuberculosis/Mycobacteriology Branch, Division of AIDS, STD, and TB Laboratory Research, Centers for Disease Control and Prevention. His research interests include application of molecular methods to epidemiology and diagnostics of mycobacterial diseases.

ack T. Crawford,

Guest Editor

1 tuberculosis has been used for more than a decade to study the epidemiology of tuberculosis (TB). DNA fingerprinting, with IS6110 as a probe, has been used successfully to trace transmission of $M$. tuberculosis in outbreaks, confirm laboratory cross-contamination, and identify risk factors for disease among populations of patients with TB (1). Before 1993, epidemiologic studies that used molecular characterization of M. tuberculosis were focused on populations, such as persons who were HIV positive or were in nosocomial settings (2-4). The standardization of methods provided an opportunity to examine $M$. tuberculosis strains from TB patients throughout the world and encompassed a variety of settings and populations (5).

In response to the upsurge of TB cases in the United States, the Centers for Disease Control and Prevention (CDC) funded regional laboratories to provide fingerprinting services to support TB control programs in outbreak investigations and to conduct studies on using DNA fingerprinting in TB epidemiology and control. This network was expanded to include sentinel surveillance sampling in 1996, when CDC established the National Tuberculosis Genotyping and Surveillance Network as a 5-year project. The genotyping network involved seven

${ }^{*}$ Centers for Disease Control and Prevention, Atlanta, Georgia, USA sentinel surveillance sites ${ }^{1}$ that were paired with seven genotyping laboratories. ${ }^{2}$ The historical background and objectives of the genotyping network have been discussed elsewhere (6). In this paper, we summarize the specific project methods.

\section{Network Participants}

The sentinel surveillance sites included the states of Arkansas, Maryland, Massachusetts, Michigan, and New Jersey; six counties in California surrounding San Francisco (Alameda, Contra Costa, Marin, San Mateo, Santa Clara, and Solano); and four counties in Texas representing two regions of the state with distinct demographics (Dallas, Tarrant, Cam-

${ }^{1}$ Sentinel surveillance sites and personnel: 1) Arkansas Department of Health: Joseph Bates, Donald Cunningham, Kashef ljaz, Bill Starrett, William Stead, and Frank Wilson; 2) California Department of Health Services: Rocio Agraz, Dan Chin, Jennifer Flood, Peter Oh, Sarah Royce, and Sumi Sun; 3) Maryland Department of Health and Mental Hygiene: Wendy Cronin, Jonathan Golub, David Hooper, Nancy Hooper, Monica Lathan, Leonard Mukasa, and Jafar Razeq; 4) Massachusetts Department of Public Health: Edward Corkren, Paul Elvin, Sue Etkind, Ann Miller, Edward Nardell, Jill Northrup, Alissa Scharf, Sharon Sharnprapai, Alexander Sloutsky, Robert Suruki, and Debra Thimas; 5) Michigan Department of Community Health: Bernie Benecke, Matthew Boulton, Norm Keon, Michael Kucab, Dennis Minnice, Jolynn Montgomery, and Charles Williams II; 6) New Jersey Department of Health and Senior Services: Nancy Buono, Mary DilIon, Zary Liu, Marcia Localio, Christian Nwigwe, Felicidad Santos, Mary Ellen Schulman, and Kenneth Shilkret; 7) Texas Department of Health: Dennis Ashworth, Dale Dingley Jr., Miguel Garza, Jeff Taylor, and Patricia Thickstun.

${ }^{2}$ Genotyping laboratories and personnel: 1) Alabama Department of Public Health and University of Alabama, Birmingham: William Benjamin Jr., Nancy Dunlap, Donna Hafner, Nancy Keenan, Kerry Lok, Donna Mulcahy, Virginia Pruitt, and Nancy Robinson; 2) Central Arkansas Veterans Healthcare Services: M. Donald Cave, Kathleen Eisenach, Ndingsa Fomukong, and ZhenhuaYang; 3) California Department of Health Services: Jason Coloma, Jonna De Leon, Edward Desmond, Athena Fye, Marguerite Griffith, Melvin Javonillo, Travis Jobe, Rose Longoria, Cynthia Sanders, and Lourdes Seli; 4) Michigan Department of Community Health: Dale Berry, Steve Church, Stephen Dietrich, Frances Pouch Downes, Jeffrey Massey, Teresa Miller, Laura Mosher, and Patricia Somsel; 5) New York State Health Department,Wadsworth Center: Jeff Driscoll, Robert Jovall, Mike McGarry, Max Salfinger, and Harry Taber; 6) Public Health Research Institute, New York: Pablo Bifani, Barry Kreiswirth, Natalia Kurepina, Barun Mathema, Soraya Moghazeh, Alex Ravikovitch, and Elena Shashkina; and 7) Texas Center for Infectious Diseases: Yvonne Camarce, Rebecca Cox, J. Seb Gillette, Oscar Gonzalez, Kenneth Jost, D. Mitch Magee, Teresa Quitugua, Ishmael I. Rosas, and Jeffery Taylor. 
eron, and Hidalgo) (Figure). State departments of health TBcontrol programs conducted the project activities for each site. The seven laboratories provided DNA fingerprint analyses of M. tuberculosis isolates for their sentinel surveillance sites. In addition, genotyping network laboratories were assigned regions for which they provided genotype analysis of $M$. tuberculosis isolates in support of investigations by state departments of health. These isolates and associated patients were not included in the network databases. Regions were delineated on the basis of the approximate number of patients with TB in the regions; regions did not follow the boundaries of the U.S. Public Health Service Regions.

\section{Case Finding and Isolate Collection}

Sources of information to identify TB cases within surveillance sites included hospital and clinic records from all facilities serving patients in the area, records of laboratories performing mycobacteriology services, hospital ICD-9CM discharge codes for TB, pharmacy records for prescriptions of anti-TB drugs, medical examiners' or coroners' records, death certificates, and AIDS surveillance reports that listed a diagnosis of TB. Culture-positive, verified TB cases reported from January 1996 through December 2000 for national TB surveillance to CDC (reported on the Report of a Verified Case of Tuberculosis) were included as sentinel surveillance cases. Patients with recurrent TB occurring $>1$ year after completion of therapy are considered new case-patients; a small number of such cases were included. Patients later identified as residing outside of the surveillance area and those with positive cultures as the result of laboratory cross-contamination were excluded.

Every effort was made to acquire an isolate from each study patient. This task was difficult because many clinical samples were sent for culturing to laboratories other than the state public health laboratory, including hospital and commercial laboratories, some out of state. Isolates identified as $M$. tuberculosis complex, i.e., M. tuberculosis, M. bovis, and M. africanum, were included in the study. Isolates were sent to the assigned genotyping laboratory for typing. Stocks of all isolates were maintained at $-70^{\circ} \mathrm{C}$, and all isolates were transferred to $\mathrm{CDC}$ for long-term storage and future studies.

\section{Laboratory Protocols}

All National Tuberculosis Genotyping and Surveillance Network laboratories used standardized protocols. Isolates were typed by the international standard IS 6110 method, i.e., digestion of DNA with endonuclease $P v u \mathrm{II}$ and hybridization with a probe containing the right end of IS6110 (5). Growth was harvested directly from Lowenstein-Jensen slants, or isolates were subcultured in Middlebrook $7 \mathrm{H} 9$ broth. Cell suspensions were heat-killed before DNA was extracted. DNA was digested with $P v u I I$ and run on a $20-\mathrm{cm}, 1 \%$ agarose gel without ethidium bromide. Electrophoresis was conducted at $100 \mathrm{~V}$ for a short time to allow the DNA to enter the gel and then overnight at lower voltage until the dye front had run

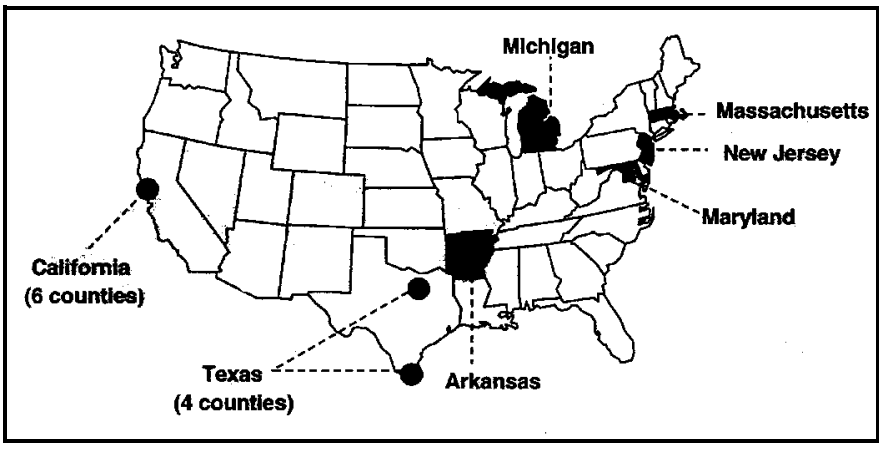

Figure. Map showing locations of the sentinel surveillance sites in the National Tuberculosis Genotyping and Surveillance Network, United States.

approximately $17 \mathrm{~cm}$. An external size standard was run in wells 1,10 , and 20 on a 20 -well gel. The gel was stained with ethidium bromide and photographed on a UV transilluminator to check for quality, including complete digestion of the DNA samples, degradation of DNA, and uneven running or smiling of the gel. Gels were blotted and hybridized in the standard fashion with chemiluminescent probes (ECL kit, Amersham Biosciences, Piscataway, NJ) and x-ray film, with the exception of one laboratory that used ${ }^{32} \mathrm{P}$-labeled probes and a phosphoimager to generate direct digital images.

Two different size standards were used. Initially, total genomic DNA from M. tuberculosis strain 2650, provided by the genotyping laboratory at the Public Health Research Institute, New York, was used. This standard was replaced by a recombinant size standard prepared at CDC; this standard contains 25 fragments of known sequences, ranging in size from 700 to $15,000 \mathrm{bp}$. All fragments contain at least one copy of a 500-bp segment of the right side of IS6110 and are detected by the IS6110 probe. When the recombinant standard was available, the sizes of the fragments in the original 2650 standard were determined relative to the new standard, and these sizes were used to reanalyze all of the prior images containing the 2650 standard.

\section{Computer-Assisted Pattern Analysis}

IS6110 patterns were scanned and then analyzed by regional laboratory personnel by using Biolmage Whole Band Analyzer software, version 3.4 (BioImage, Ann Arbor, MI), a UNIX-based program run on Sun workstations (Sun Microsystems, Inc., Santa Clara, CA). This version of the software included enhancements developed specifically for the TB genotyping network. The software performed automatic band identification, but each image was visually evaluated and edited, as needed. The greatest difficulty was deciding if bands of greater than average width or intensity represented single or multiple fragments. The protocol called for assigning multiple bands only when separate bands were clearly evident, which often required observation of multiple exposures of the original films. Bands with lower than average intensity were counted only if the peak height in the scanned image was at least two thirds that of adjacent bands. The logarithmic scale method was used to size bands. 
For pattern matching, BioImage software used the Jaccard coefficient of similarity between two patterns, A and B (100 x number of matched bands [number of bands in $\mathrm{A}+$ number of bands in B - number of matched bands]). Pairs of patterns were compared for matching bands by a deviation of $\pm 2.5 \%$ for molecular weight (not distance migrated). All matches were verified by visual comparisons. Only patterns that were identical, i.e., had the same number of bands of the same sizes $( \pm 2.5 \%)$, were given the same designation.

Each regional laboratory maintained a database of fingerprint patterns for all isolates from the laboratory's sentinel site. New patterns were submitted to CDC for assignment of a genotyping network database designation. Any subsequent isolates with the identical fingerprint pattern were assigned the same designation and were not submitted to CDC. Files of single gel lanes were transmitted to a secure site at CDC. These files contained the original scanned image and the data file of calculated band sizes. In these images, bands included in the analysis were highlighted, and the locations of the bands in the standard were indicated. The database manager examined the images to determine if they were of acceptable quality but did not make changes in the submitted files. Apparent discrepancies were referred to the submitting laboratory for resolution, and the final decision about band identification rested with the laboratory that performed the analysis and had the original films. Submitted patterns were compared to the patterns in the existing TB genotyping network database by using the parameters described above. New patterns were added to the database and assigned consecutive 5-digit designations. When a submitted pattern matched a pattern already in the database, the submitted pattern was reported back with the existing designation. Thus, the TB genotyping network fingerprint database contained single examples of all distinct patterns from all seven regional laboratories during the 5 -year study period. The designation 00000 was assigned to isolates having zero copies of IS6110, i.e., gave no bands. Some patterns were dropped from the study, e.g., if the isolate was the result of cross-contamination or the patient was from outside the study area. These designations were not reused; therefore, the total number of patterns $(6,128)$ in the final database is lower than the highest designation (pattern 07193).

\section{Secondary Typing}

During the early part of the study, polymorphic GC-rich sequence (PGRS) typing, also referred to as pTBN12 (7), was used for secondary typing. However, this method was difficult to standardize, and spoligotyping (spacer oligotyping) was used for secondary typing (8). Isolates with six or fewer bands on IS6110 typing, including most isolates that had been typed previously by using PGRS, were spoligotyped by using a standard method (9). The spoligotypes were recorded by using a 15-digit octal code to represent the binary result for the 43 spacers (10). Briefly, the 43-digit binary result, representing the 43 spacers (where $1=$ positive, $0=$ negative), was divided into 14 sets of 3 digits (spacers $1-42$ ) plus 1 additional digit (spacer 43). Each 3-digit set was converted to octal code $(000=0,001=1,010=2,011=3,100=4,101=5,110=6$, and $111=7$ ) with the final digit remaining either 1 or 0 . This scheme yielded a 15-digit octal designation. To simplify database entries, the CDC database manager assigned consecutive arbitrary 4-digit designations to the octal designations.

\section{Quality Assurance}

Several sets of isolates were prepared at CDC and distributed to the typing laboratories for fingerprinting and analysis. Their results and results of typing at $\mathrm{CDC}$ were analyzed to determine reproducibility. Because such challenge sets may receive special handling, a second approach was used in the final 3 years of the project. For each laboratory, the database manager selected IS6110 patterns at random from those previously submitted by that laboratory to the CDC database. The corresponding isolates, 10 per year per laboratory, were sent to CDC for typing, and results were compared to prior patterns.

\section{Epidemiologic Databases}

Sentinel surveillance sites each maintained a database of their patients and patient isolate genotype designations in EpiInfo $6.04 \mathrm{~d}$ software (case file) (11). Patients were identified by their state surveillance case numbers. Complete files from each sentinel site were transmitted to CDC on a bimonthly basis and concatenated. These data were merged with select variables from the national TB surveillance database (SURVSTB and TIMS) in an SAS dataset (SAS Institute, Inc., Cary, NC).

For each sentinel surveillance patient, data were collected concerning any TB case-patients identified as epidemiologically related through routine contact investigations conducted as part of TB-control programs. Epidemiologic connections were defined as direct exposure to an infectious TB patient (e.g., named contacts) or a circumstance in which patients were in the same location at the same time (e.g., the same jail). Related cases identified though routine contact investigations were included in the study if they had been diagnosed on or after January 1, 1990. Data collected for each related casepatient included the following: direction of transmission (source, secondary); type of relationship (household, nonhousehold relatives or friends, co-worker, common source); type of setting of exposure (e.g., correctional facility, school or day-care center, co-worker, emergency shelter, group quarters, hospital, nursing home, other long-term care facility); and date that the epidemiologic relationship was discovered. Information for each related case identified through routine contact investigation was entered into a supplemental Epi-Info file, which was also routinely submitted to CDC. This database was merged with the case file data in an SAS dataset.

\section{Genotype Cluster Investigation}

Case-patients with isolates demonstrating indistinguishable genotypes, i.e., the same IS6110-pattern designation for isolates with more than six IS6110 bands or the same IS6110 
pattern and spoligotype for isolates with six or fewer IS6110 bands, were defined as genotype clusters. All cases in clusters required review of epidemiologic information obtained from contact investigations before isolate genotyping to determine known epidemiologic connections. If one or more patients in a cluster had no known epidemiologic connections with others in the cluster, a cluster investigation was initiated. Patients participated with informed consent. Cluster investigations were conducted prospectively from January 1, 1998, to December 31, 2000. Data from medical record reviews and interviews with the patients were collected by using standardized forms. Information collected from interviews included demographics, TB medical history, any TB exposures, and at least a 2-year history for residence and social, work, and recreational activities. Information collected from medical records and interviews was compared for patients in each cluster to identify epidemiologic connections. The results of cluster investigations were entered into a third Epi-Info file. This file also contained related positive skin-test results, date of initial skin tests, direction of transmission, types of relationships, exposure settings, start dates of likely exposure, and information used to identify relationships (record review, routine interview, contact investigation, and isolate genotyping information). Data were submitted to CDC and converted into an SAS dataset for future analyses.

\section{Weaknesses in Study Design}

From the beginning of the project, we recognized that compiling results from multiple laboratories would be challenging because of the variability in the IS6110 fingerprinting method. However, assigning a designation to the IS6110 pattern for each isolate was necessary to allow entry of the result into the epidemiologic database. Two flaws in the design of the national fingerprint database were identified during the project; both primarily affected the analysis of isolates with low-copy numbers for IS6110. The first flaw arose from the computer algorithm for pattern matching. A newly submitted pattern (B) was considered distinct from an existing pattern (A) if any band in the pattern differed by more than the allowed $2.5 \%$ variation in size. However, a third pattern with a band of intermediate size can match both A and B. Thus, the software could identify a set of patterns that matched at $100 \%$, even though some individual pairs within the set did not match. The second flaw was procedural. When a laboratory submitted a pattern that matched one already in the database, the existing designation was reported back to that laboratory. In such situations, the submitted image became the reference in that laboratory's database for that pattern designation. In retrospect, we realize that more consistent results would have been obtained if we had transmitted the prototype image from the national database to the regional laboratories so that everyone would have been using the same patterns for matching, i.e., the same set of band sizes.

Quality assurance results (12) highlighted the need for experienced judgment in evaluating and editing patterns and matching results. In addition, isolates with few copies of IS6110 and common spoligotypes may not be well discriminated with these genotyping methods. The subjective nature of genotype interpretation and lack of specificity for some isolates may have resulted in some patients being misclassified as clustered or not.

Determination of epidemiologic connections based on routine contact investigations was problematic. Methods used by different jurisdictions varied considerably, as did the completeness of contact investigations. Therefore, interpretation and comparison of the proportion of cases with epidemiologic connections established by contact investigations among the sentinel sites should be done with caution. Genotype cluster investigations proved particularly difficult in several situations. Though cluster investigations were conducted prospectively, some genotype results were delayed for prolonged periods. Patients who had completed therapy were often difficult to locate for interviews. Delay in identifying clusters also occurred when a prolonged period had elapsed between diagnoses and cultures of patient specimens; delays also varied among the sites. The success of identifying epidemiologic links among clustered patients in these circumstances was limited.

\section{Acknowledgments}

We thank Charles Woodley and Lauren Cowan for their invaluable laboratory support and Ken Castro, Harold Jaffe, and Thomas Shinnick for their scientific guidance.

\section{References}

1. Small PM, van Embden JDA. Molecular epidemiology of tuberculosis. In: Bloom B, editor. Tuberculosis: pathogenesis, protection, and control. Washington: American Society for Microbiology; 1994. p. 569-82.

2. Edlin BR, Tokars JI, Grieco MH, Crawford JT, Williams EM, Sordillo $\mathrm{KR}$, et al. An outbreak of multi-drug resistant tuberculosis among hospitalized patients with the acquired immunodeficiency syndrome. N Engl J Med 1992;326:1514-21.

3. Pearson ML, Jereb JA, Freiden TR, Crawford JT, Davis BJ, Dooley SW, et al. Nosocomial transmission of multi-drug resistant Mycobacterium tuberculosis: a risk to patients and healthcare workers. Ann Intern Med 1992;117:191-6.

4. Beck-Sague C, Dooley SW, Hutton MD, Otten J, Breeden A, Crawford JT, et al. Hospital outbreak of multi-drug resistant Mycobacterium tuberculosis infections: factors in transmission to staff and HIV-infected patients. JAMA 1992;268:1280-6.

5. van Embden JDA, Cave MD, Crawford JT, Dale JW, Eisenach KD, Gicquel B, et al. Strain identification of Mycobacterium tuberculosis by DNA fingerprinting: recommendations for a standardized methodology. J Clin Microbiol 1993;31:406-9.

6. Castro KG, Jaffe HW. Rationale and methods for the National Tuberculosis Genotyping and Surveillance network. Emerg Infect Dis 2002; 8;1188-91.

7. Ross BC, Raios K, Jackson K, Dwyer B. Molecular cloning of a highly repeated DNA element from $M$. tuberculosis and its use as an epidemiological tool. J Clin Microbiol 1992;30:942-6.

8. Kamerbeek J, Schouls L, Kolk A, van Agterveld D, van Soolingen D, Kuijpers S, et al. Simultaneous detection and strain differentiation of Mycobacterium tuberculosis for diagnosis and epidemiology. J Clin Microbiol 1997;35:907-14. 
9. Molhuizen HO, Bunschoten AE, Schouls LM, van Embden JDA. Rapid detection and simultaneous strain differentiation of Mycobacterium tuberculosis complex bacteria by spoligotyping. In: Parish T, Stoker NG, editors. Mycobacterial protocols. Totowa (NJ): Humana Press; 1998. p. 381-94.

10. Dale JW, Brittain D, Cataldi AA, Cousins D, Crawford JT, Driscoll J, et al. Spacer oligonucleotide typing of bacteria of the Mycobacterium tuberculosis complex: recommendations for standard nomenclature. Int $\mathrm{J}$ Tuberc Lung Dis 2001;5:216-9.

All material published in Emerging Infectious Diseases is in the public domain and may be used and reprinted without special permission; proper citation, however, is appreciated.
11. Dean AG, Dean JA, Coulombier D, Burton AH, Brendel KA, Smith DC, et al. Epi-Info, Version 6.04a, a word processing, database, and statistics program for public health on IBM-compatible microcomputers. Atlanta: Centers for Disease Control and Prevention; 1996.

12. Braden CR, Crawford JT, Schable BA. Quality assessment of Mycobacterium tuberculosis genotyping in a large laboratory network. Emerg Infect Dis $2002 ; 8 ; 1210-5$.

Address for correspondence: Jack T. Crawford, Centers for Disease Control and Prevention, Mailstop F08, 1600 Clifton Rd., N.E., Atlanta, GA 30333, USA; fax: 404-639-1287; e-mail: jcrawford@cdc.gov
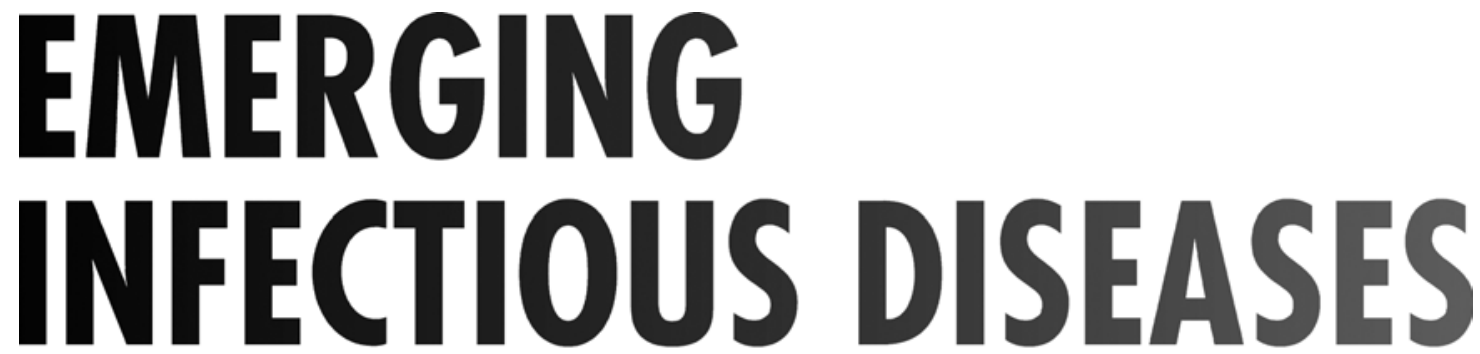

A Peer-Reviewed Journal Tracking and Analyzing Disease Trends

\section{Detailed Instructions for Emerging Infectious Diseases Authors}

\section{Types of Articles}

Perspectives. Articles should be under 3,500 words and should include references, not to exceed 40 . Use of subheadings in the main body of the text is recommended. Photographs and illustrations are encouraged. Provide a short abstract (150 words) and a brief biographical sketch of first author. Articles in this section should provide insightful analysis and commentary about new and reemerging infectious diseases and related issues. Perspectives may also address factors known to influence the emergence of diseases, including microbial adaptation and change, human demographics and behavior, technology and industry, economic development and land use, international travel and commerce, and the breakdown of public health measures. If detailed methods are included, a separate section on experimental procedures should immediately follow the body of the text.

Synopses. Articles should be under 3,500 words and should include references, not to exceed 40 . Use of subheadings in the main body of the text is recommended. Photographs and illustrations are encouraged. Provide a short abstract ( 150 words) and a brief biographical sketch of first author-both authors if only two. This section comprises concise reviews of infectious diseases or closely related topics. Preference is given to reviews of new and emerging diseases; however, timely updates of other diseases or topics are also welcome. If detailed methods are included, a separate section on experimental procedures should immediately follow the body of the text.

Research Studies. Articles should be under 3,500 words and should include references, not to exceed 40 . Use of subheadings in the main body of the text is recommended. Photographs and illustrations are encouraged. Provide a short abstract (150 words) and a brief biographical sketch of first author-both authors if only two. Report laboratory and epidemiologic results within a public health perspective. Although these reports may be written in the style of traditional research articles, they should explain the value of the research in public health terms and place the findings in a larger perspective (i.e., "Here is what we found, and here is what the findings mean").

Policy and Historical Reviews. Articles should be under 3,500 words and should include references, not to exceed 40 . Use of subheadings in the main body of the text is recommended. Photographs and illustrations are encouraged. Provide a short abstract (150 words) and a brief biographical sketch.
Articles in this section include public health policy or historical reports that are based on research and analysis of emerging disease issues.

Dispatches. Articles should be 1,000-1,500 words and need not be divided into sections. If subheadings are used, they should be general, e.g., "The Study" and "Conclusions." Provide a brief abstract (50 words); references (not to exceed 15); figures or illustrations (not to exceed two); and a brief biographical sketch of first author - both authors if only two. Dispatches are updates on infectious disease trends and research. The articles include descriptions of new methods for detecting, characterizing, or subtyping new or reemerging pathogens. Developments in antimicrobial drugs, vaccines, or infectious disease prevention or elimination programs are appropriate. Case reports are also welcome.

Commentary. Thoughtful discussions (500-1,000 words) of current topics. Commentaries may contain references but should not include figures or tables.

Another Dimension. Thoughtful essays, short stories, or poems on philosophical issues related to science, medical practice, and human health. Topics may include science and the human condition, the unanticipated side of epidemic investigations, or how people perceive and cope with infection and illness. This section is intended to evoke compassion for human suffering and to expand the science reader's literary scope. Manuscripts are selected for publication as much for their content (the experiences they describe) as for their literary merit.

Letters. This section includes letters that present preliminary data or comment on published articles. Letters (500-1,000 words) should not be divided into sections, nor should they contain figures or tables. References (not more than 10) may be included.

Book Reviews. Short reviews (250-500 words) of recently published books on emerging disease issues are welcome. The name of the book, publisher, and number of pages should be included.

News and Notes. We welcome brief announcements (50-150 words) of timely events of interest to our readers. (Announcements may be posted on the journal Web page only, depending on the event date.) In this sec-tion, we also include summaries (500-1,000 words) of emerging infectious disease conferences. Summaries may provide references to a full report of conference activities and should focus on the meeting's content. 